\section{Análise de conformidades às normas técnicas de proteção radiológica dos serviços de radioterapia no Estado de São Paulo, Brasil}

\author{
Compliance with technical standards for \\ radiological protection at radiation therapy \\ services in São Paulo State, Brazil
}

Maria Bernadete de Paula Eduardo 1 Hillegonda Maria Dutilh Novaes 2

\footnotetext{
1 Centro de Vigilância Epidemiológica, Secretaria de Estado da Saúde de São Paulo, São Paulo, Brasil. 2 Faculdade de Medicina, Universidade de São Paulo, São Paulo, Brasil.

Correspondência M. B. P. Eduardo Centro de Vigilância Epidemiológica, Secretaria de Estado da Saúde de São Paulo.

Av. Dr. Arnaldo 351, 6o andar, sala 607, São Paulo, SP 01246-001, Brasil. mbpeduardo@uol.com.br
}

\section{Abstract}

Radiation therapy services provide essential therapeutic procedures for cancer, one of the main causes of population morbidity and mortality. Despite their importance in the health system and their potential risks due to the use of ionizing radiation, there are few studies on such services. We evaluated compliance with technical standards for radiological protection in radiation therapy services in São Paulo State, Brazil. Forty-nine services were studied in 2000 through interviews with technical staff. Typologies of performance profiles focusing on structure and process variables were constructed and services compared. Important differences were observed in the services' positions in the health care system, level of complexity, and geographic distribution, with better average performance in structural conditions but very inadequate performance in patient protection, indicating the need for more effective health surveillance.

Neoplams; Radiotherapy; Radiation; Health Surveillance

\section{Introdução}

Os serviços de radioterapia ocupam importante posição no SUS, no atendimento às neoplasias que, juntamente com as doenças cardiovasculares, representam um dos problemas mais graves de saúde, tanto nos países industrializados quanto nos em desenvolvimento 1. No Estado de São Paulo, a mortalidade proporcional por neoplasias malignas passou de 9,0\% em 1970 para $14,8 \%$ em 1998, alternando-se com as causas externas na segunda ou terceira causa de morte 2 , constituindo-se em um importante fator para o crescimento do setor de radioterapia na década de 90 na região, com mais de 60,0\% desses serviços no Brasil 3. Estimativas para o ano de 19984 apontavam a ocorrência de em torno de 269 mil novos casos e 107.905 óbitos no Brasil, com 33.132 óbitos no Estado de São Paulo, isto é, cerca de 30,0\% dos óbitos por neoplasias no país 2 .

Outra questão importante nos serviços de radioterapia é o risco envolvido em seus procedimentos - o uso da radiação para fins médicos - fazendo com que o setor esteja submetido a uma dupla regulação sanitária, o controle da Comissão Nacional de Energia Nuclear (CNEN) e da Vigilância Sanitária, por meio da Agência Nacional de Vigilância Sanitária (ANVISA) em nível federal e equipes de secretarias de saúde estaduais e municipais, em níveis regional e local. Face aos perigos potenciais, trata-se de uma 
área profícua em normas técnicas e legislação, sendo regida por princípios inclusive internacionais, facilitando o desenvolvimento de indicadores de monitoramento dos serviços.

Os serviços de radioterapia compreendem as especialidades de teleterapia (uso de radiação emitida por bombas de cobalto, aceleradores lineares e equipamentos convencionais de raios $\mathrm{X}$ ), braquiterapia (uso de radiação à curta distância ou com implantação de fontes de radiação de baixa ou alta dose no paciente) e betaterapia (fontes de baixa energia utilizadas em procedimentos superficiais). Aceleradores lineares e equipamentos de braquiterapia de alta dose, bem como equipamentos computadorizados para o planejamento de tratamento e simuladores, são considerados desenvolvimentos tecnológicos que aumentam a precisão das aplicações e qualidade, segurança e efetividade dos tratamentos, além de permitir uma otimização do trabalho do médico 1,5,6.

Apesar de sua importância para a prática clínica e de seu potencial risco aos usuários, os serviços de radioterapia são ainda pouco estudados, particularmente no Brasil e América Latina. Há uma importante produção internacional de avaliação de programas de garantia de qualidade em radioterapia centrada em aspectos dos procedimentos médicos relacionados ao tratamento de câncer, como qualidade e eficácia das doses de radiação, eventos adversos e riscos 7,8,9,10,11, desvios de doses e prognósticos 12, manuais técnicos, legislação ou recomendações produzidas por órgãos reguladores e comitês científicos de diversos países 13,14 15,16,17. Também são encontradas avaliações que abordam a radioterapia como uma das condutas entre as alternativas terapêuticas para o câncer ou as políticas adotadas na organização da atenção e dos serviços, no que diz respeito ao acesso e demanda dos pacientes, eqüidade na atenção e utilização de avaliações econômicas 18,19,20,21,22,23. Contudo, são ainda escassos na literatura internacional os estudos em serviços específicos e que enfoquem o cumprimento dos padrões propostos de segurança radiológica.

A literatura nacional restringe-se à discussão de protocolos de programas de garantia de qualidade 24 , manuais técnicos ou recomendações para as especialidades em radioterapia 25,26,27,28 e estudos de alternativas terapêuticas 29,30. Destaca-se o trabalho de Zanardo 31, que estudou serviços de radioterapia no Brasil, na ótica da economia da saúde. Pesquisas em nível nacional sobre proteção radiológica nos ser- viços de radioterapia são raros, destacando-se um estudo de exposição ocupacional em serviços médicos que utilizam radiação ionizante desenvolvido por Drexler 32.

As avaliações de programas e de qualidade em serviços de saúde, compreendidas como análises de dimensões estruturais, de processos ou resultados objetivando o desenvolvimento de um juízo de valor, apresentaram um notável crescimento nas últimas décadas, e se constituem em práticas apoiadas em metodologias de natureza quantitativa e qualitativa diversas, selecionadas de acordo com o contexto no qual elas se desenvolvem e os objetivos a serem alcançados 33,34,35,36,37,38.

O estudo aqui apresentado teve como objetivo identificar as características dos serviços de radioterapia e avaliar as suas conformidades às normas técnicas e legislação referentes à proteção radiológica dos pacientes, trabalhadores e público nos serviços, utilizando variáveis de estrutura e processo consideradas mais adequadas para as dimensões a serem estudadas no programa. Os indicadores de desempenho do programa analisado neste estudo são os indicadores de conformidades ao arcabouço normativo específico, e foram construídos apoiados nas literaturas nacional e internacional e normas e legislação que regulam o setor no Brasil e no Estado de São Paulo. Variáveis de resultado, como a efetividade dos tratamentos realizados, não foram incluídos no estudo, e no que diz respeito à qualidade da atenção ao paciente esta se restringiu à avaliação da aplicação dos procedimentos relativos à minimização dos riscos para o mesmo da exposição radiológica.

\section{Metodologia}

\section{Desenho da pesquisa e universo do estudo}

A pesquisa exigiu, de início, uma análise dos documentos programáticos e normativos, relatórios técnicos e legislação dos órgãos reguladores para a identificação do enfoque conceitual que norteia o controle sanitário no setor de radioterapia em níveis nacional e estadual, dos objetivos e metas dos programas de vigilância sanitária, bem como das ferramentas utilizadas nas inspeções sanitárias, para a identificação de suas formas de atuação, indicadores utilizados e aspectos priorizados nas fiscalizações. 
Apoiados nos enunciados programáticos e normativos que regulam o setor, foi construído o modelo que orientou o desenvolvimento da pesquisa empírica, levando à construção de indicadores de avaliação das conformidades às normas de proteção radiológica, permitindo a avaliação do controle sanitário nessa dimensão. Foram também utilizadas na seleção dos indicadores e elaboração dos questionários as recomendações sobre padrões de referência aprovadas pelo American National Standards Institute (ANSI) 37 e pelo Joint Committee on Standards for Educational Evaluation (JCSEE) 39.

A coleta de dados foi realizada no período de janeiro a novembro de 2000, com a utilização de questionário semi-estruturado em entrevistas com diretores clínicos ou supervisores de radioproteção dos serviços de radioterapia. Participaram do estudo 50 dos 54 serviços de radioterapia em funcionamento no Estado de São Paulo, identificados pelo Cadastro de Serviços de Radioterapia da CNEN 3. Quatro serviços se recusaram a participar do estudo. Um serviço, considerado pelos especialistas na área como sendo de referência na excelência técnica, não foi incluído na consolidação geral dos dados ao ter se constituído em referência para a definição dos quesitos e critérios, ter contribuído para a elaboração dos instrumentos a serem aplicados no que diz respeito à compreensão das questões, colaborado no aperfeiçoamento e validação dos mesmos, bem como, nas definições das tipologias de desempenho dos serviços.

O questionário foi também discutido com especialistas do setor de radioterapia e vigilância sanitária. Quinze equipes de vigilância sanitária de nível estadual e três de nível municipal, dentre o total de 24 equipes com serviços de radioterapia na sua área de abrangência, participaram do estudo com informações complementares sobre os serviços inspecionados por elas e na comparação das informações fornecidas pelos serviços e a situação identificada pela vigilância sanitária.

\section{Fontes de dados}

As informações utilizadas na pesquisa foram obtidas com base em várias fontes:

(a) Consulta a documentos oficiais da Secretaria de Estado da Saúde do Estado de São Paulo (SES-SP) e entrevistas com profissionais da coordenação da vigilância sanitária estadual: para obtenção de informações sobre recursos humanos, caracterização da complexidade tecnológica e posição no sistema de saúde dos serviços de radioterapia. (b) Entrevistas com profissionais responsáveis pelos serviços: para as informações sobre as conformidades à regulação sobre proteção radiológica e caracterização dos recursos humanos e materiais, complexidade tecnológica e posição no sistema de saúde.

(c) Questionários preenchidos pelas equipes regionais e municipais de vigilância sanitária com dados das inspeções realizadas nos serviços de radioterapia.

(d) Acesso a sistemas de informação em saúde para coleta de dados sobre morbidade hospitalar (Sistema de Informações Hospitalares/Departamento de Informática do SUS - SIH/DATASUS), mortalidade por câncer (Sistema de Informações sobre Mortalidade - SIM/DATASUS), estimativas de morbidade e mortalidade por câncer (Instituto Nacional do Câncer - INCA), procedimentos de alta complexidade (Sistema de Informações Ambulatoriais/DATASUS) e cadastros dos órgãos reguladores (CNEN, ANVISA e Centro de Vigilância Sanitária/SES-SP).

O profissional entrevistado nos serviços, além de responder às questões, conduzia o pesquisador para uma visita pelo serviço. Aqueles serviços que recusaram a entrevista foram incluídos apenas no quadro de caracterização geral do setor, baseado em outras fontes de informação. Em três serviços o questionário foi preenchido à distância, tendo sido anexados aos questionários fotos e cópias de documentos comprobatórios das informações registradas.

\section{Indicadores para avaliação \\ das conformidades}

Os indicadores para avaliação das conformidades foram desenvolvidos com base nos princípios de proteção radiológica no uso da radiação ionizante em pacientes, trabalhadores e público, e nos de controle e garantia de qualidade. A seleção dos indicadores teve como referência os quesitos estabelecidos: (1) pela legislação e normas técnicas vigentes $3,40,41,42,43,44,45,46$; (2) pelas normas de credenciamento dos serviços ao SUS 47,48; (3) em parâmetros técnicos referidos pelas literaturas nacional e internacional sobre segurança e qualidade no setor 1,5,6,24,49.

Foram construídos quatro grupos de conformidades por meio dos quais foi mensurado o desempenho dos serviços de radioterapia:

- Grupo I - Documentação: documentos emitidos pelos órgãos reguladores que atestam a conformidade da estrutura física a padrões técnicos de segurança e qualidade, condições para a sua construção e autorização de funcionamento (tais como: alvará da vigilância sanitária, licença da CNEN para construção e apro- 
vação do projeto, certificados de especialização de recursos humanos e registros nos conselhos, plano de proteção radiológica, sistemas de segurança e emergência, programas de monitoramento da radiação individual e do meio ambiente, programas de treinamento);

- Grupo II - Proteção radiológica de público/vizinhanças: enquadramento nas exigências legais e condições técnicas do serviço necessárias para operar dentro dos princípios de segurança para o público e vizinhanças;

- Grupo III - Proteção radiológica dos trabalhadores: enquadramento nas exigências legais quanto às condições de segurança dos trabalhadores expostos às radiações ionizantes;

- Grupo IV - Proteção radiológica do paciente: cuidados de segurança e de qualidade nos procedimentos que minimizam as exposições à radiação e risco de danos e lesões.

Cada grupo foi composto por itens de conformidades e estes por perguntas, com escores para cada uma delas com valor de 0 a 2 , indicando o cumprimento total ou parcial, ou não cumprimento, daquele quesito. A taxa de conformidade para cada item foi obtida com a soma dos escores das perguntas, transformada em proporção percentual em relação aos pontos brutos máximos possíveis, representando assim um percentual de alcance do item de conformidades. Cada grupo de conformidades foi representado por uma média aritmética calculada com base nos percentuais obtidos nos respectivos itens. Como os grupos de conformidades têm números diversos de perguntas e itens, não se mostrou adequado utilizar na sua caracterização o total de pontos obtidos para cada conjunto, e decidiu-se pela utilização das médias das proporções de conformidades encontradas, inclusive para o cálculo da média final que qualificou os serviços em conformidades Alta, Média ou Baixa. Os grupos e itens de conformidades com seus respectivos escores estão referidos na Tabela 1.

\section{O questionário para as entrevistas}

O questionário utilizado nas entrevistas nos serviços incluiu questões abertas e fechadas relativas às dimensões de estrutura e processo, necessárias à construção dos indicadores. Foram incluídas também perguntas relativas à caracterização geral dos estabelecimentos: recursos humanos, tipo e quantidade de equipamentos, especialidades oferecidas, formas de inserção no sistema de atenção à saúde, formas de financiamento. Foram obtidas informações sobre as fiscalizações realizadas pela CNEN e Vigilância Sanitária, e tipos de infra- ções constatadas e opiniões sobre os órgãos fiscalizadores, dados não incluídos nos cálculos dos escores, mas que permitiram o desenvolvimento de análises adicionais não apresentadas neste texto.

\section{Análise desenvolvida}

Os serviços de radioterapia foram classificados segundo: (1) região: Grande São Paulo - R ou Interior - I; (2) condição: unidade autônoma - A ou vinculada a hospital - H; (3) complexidade tecnológica: baixa - B, média - $\mathrm{M}$ ou alta - A; e (4) entidade mantenedora. Os três primeiros itens, na ordem apresentada, compuseram o código de identificação do serviço, seguido de numeração aleatória de 1 a 49, para efeito de apresentação dos dados.

Para a classificação dos serviços segundo sua complexidade tecnológica foram utilizados vários critérios, sendo considerados de Baixa complexidade os que utilizam somente baixa energia, como na betaterapia e máquinas convencionais de raios X; Média complexidade os serviços com máquinas de cobalto 60 e de baixa energia; e Alta complexidade os com pelo menos um acelerador linear, podendo realizar todos os outros tipos de especialidades com máquinas de alta ou baixa energia.

\section{Resultados e discussão}

\section{Características gerais dos serviços de radioterapia}

Dos 54 serviços de radioterapia no Estado de São Paulo, 22 se concentravam na região da Grande São Paulo $(40,7 \%)$ e 27 no interior (59,3\%). Nesses serviços, 46 desenvolviam especialidades em teleterapia e braquiterapia, sendo $37(80,4 \%)$ vinculados ao SUS. Dos 31 serviços de Alta complexidade, 17 (54,8\%) localizavam-se na Grande São Paulo e $14(45,2 \%)$ no interior. Dos 15 de Média complexidade, 13 estavam no interior e dos oito serviços de $\mathrm{Bai}$ xa complexidade, cinco estavam no interior.

Quanto à entidade mantenedora, entre os 31 serviços de Alta complexidade, 19 (61,3\%) eram filantrópicos e/ou privados com convênio SUS, 7 (22,6\%) filantrópicos e/ou privados não conveniados ao SUS, 4 (12,9\%) públicos estaduais e $1(3,2 \%)$ público federal. Entre os 15 de Média complexidade, 10 (66,6\%) eram filantrópicos e/ou privados com convênio SUS, 1 privado não conveniado ao SUS, $3(20,0 \%)$ públicos estaduais e 1 público municipal. Dos 8 serviços de Baixa complexidade, 6 eram filan- 
Grupos e itens de conformidades* dos serviços de radioterapia - perfis de desempenho.

\begin{tabular}{|c|c|c|}
\hline Grupos e itens de conformidades* do serviço de radioterapia & $\begin{array}{c}\text { Escores brutos } \\
\text { máximos }\end{array}$ & Alcance $(\%)$ \\
\hline \multicolumn{3}{|l|}{ Grupo I - Documentação } \\
\hline Item I - plano de proteção radiológica (PPR) & 19 & 100,0 (a) \\
\hline - A - conformidade à legislação & 2 & \\
\hline - B - descrição/situação dos principais aspectos do PPR & 16 & \\
\hline - C - pertinência das opiniões sobre essas exigências legais & 1 & \\
\hline Item II - outros documentos & 14 & $100,0(b)$ \\
\hline - A - documentação legal apresentada & 5 & \\
\hline - B - documentação dos médicos responsáveis técnicos & 3 & \\
\hline - C - documentação do físico médico supervisor de radioproteção & 1 & \\
\hline - D - documentação CNEN avaliada & 2 & \\
\hline - E - documentação VISA avaliada & 2 & \\
\hline - F - pertinência das opiniões sobre as exigências legais & 1 & \\
\hline Media aritmética obtida entre as proporções & & $100,0(a+b) / 2(x i)$ \\
\hline \multicolumn{3}{|l|}{ Grupo II - Proteção radiológica de público/vizinhanças } \\
\hline Item I - instalações físicas & 3 & 100,0 (c) \\
\hline - A - em acordo com a legislação & 2 & \\
\hline - B - pertinência das opiniões sobre essas exigências legais & 1 & \\
\hline Item II - proteção radiológica das salas & 15 & $100,0(d)$ \\
\hline - A - requisitos de segurança para as salas de tratamento & 2 & \\
\hline - B - descrição/situação dos principais aspectos/cuidados & 12 & \\
\hline - C - pertinência das opiniões sobre essas exigências legais & 1 & \\
\hline Item III - programa de garantia de qualidade (PGQ) & 14 & $100,0(e)$ \\
\hline - A - número de pacientes/máquina ano & 4 & \\
\hline - B - manutenção & 1 & \\
\hline - C - PGQ: qualidade e segurança das máquinas em acordo & 2 & \\
\hline - D - descrição/situação dos principais aspectos do PGQ realizado & 6 & \\
\hline - E - pertinência das opiniões sobre as exigências legais & 1 & \\
\hline Média aritmética obtida entre as proporções & & $100,0(c+d+e) / 3(x i i)$ \\
\hline \multicolumn{3}{|l|}{ Grupo III - Proteção radiológica dos trabalhadores } \\
\hline Item I - cuidados com funcionários/trabalhadores & 10 & $100,0(f)$ \\
\hline - A - requisitos de segurança e cuidados em acordo & 2 & \\
\hline - B - descrição/situação dos aspectos de segurança dos funcionários & 7 & \\
\hline - $\mathrm{C}$ - pertinência das opiniões sobre as exigências legais & 1 & \\
\hline Média aritmética obtida entre as proporções & & $100,0(f)(x i i i)$ \\
\hline \multicolumn{3}{|l|}{ Grupo IV - Proteção radiológica do paciente } \\
\hline Item I - cuidados/garantia e qualidade com o paciente & 15 & $100,0(\mathrm{~g})$ \\
\hline - A - cuidados para redução/minimização de danos & 15 & \\
\hline Item II - procedimentos de emergência & 10 & $100,0(h)$ \\
\hline - A - medidas estabelecidas para controlar possíveis emergências & 8 & \\
\hline - B - existência de guias/manuais de procedimentos & 2 & \\
\hline Item III - preparo da equipe e suficiência & 4 & 100,0 (i) \\
\hline - A - capacitação quanto aos fundamentos básicos da radiação ionizante & 1 & \\
\hline - B - equipe capacitada para as tarefas & 1 & \\
\hline - C - suficiência da equipe (profissionais/pacientes e máquinas) & 2 & \\
\hline Média aritmética obtida entre as proporções & & $100,0(g+h+i) / 3(x i v)$ \\
\hline Média aritmética final/total obtida entre as & & $(x i+$ \\
\hline
\end{tabular}

Símbolos: letras de a a i e xi a xiv ilustram as operações matemáticas realizadas para o cálculo das médias aritméticas obtidas nos grupos e respectivos itens de conformidade, bem como, da média final.

* 96 variáveis avaliadas compõem os agrupamentos aqui apresentados como grupos e itens de conformidades.

CNEN = Comissão Nacional de Energia Nuclear; VISA = Vigilância Sanitária 
trópicos e/ou privados sem convênio SUS e 2 privados conveniados ao SUS.

Foram identificados no estudo 37 equipamentos de teleterapia com cobalto 60 , dos quais 14 no interior $(64,9 \%)$ e 39 aceleradores lineares, $19(48,7 \%)$ na capital. Os equipamentos de braquiterapia de taxa alta de dose (HDR), localizavam-se, em sua maioria, também na capital. $\mathrm{O}$ número de fontes de betaterapia foi semelhante na capital e no interior, com 14 serviços $(38,9 \%)$ na Grande São Paulo (incluindo a capital) e 22 $(61,1 \%)$ no interior. Os equipamentos de raios $\mathrm{X}$ convencionais concentraram-se no interior.

A capital, com a maior proporção de serviços de radioterapia (33,3\%), concentrou também maior quantidade de equipamentos com tecnologia mais moderna, e em especial os aceleradores lineares, que deverão substituir progressivamente os equipamentos de cobalto. Além disso, dos oito serviços com sistemas computacionais para planejamento dos tratamentos, sete localizavam-se na capital e dos serviços com simulador, cinco estavam na capital.

A média de radioterapeutas por serviço foi de 3,7 nos serviços de Alta complexidade, 1,9 nos de Média complexidade (média abaixo da recomendação oficial) e 0,6 nos de Baixa complexidade (em grande parte serviços de betaterapia em oftalmologia, sem parâmetros para avaliação). Foram computados para o conjunto dos serviços 143 radioterapeutas, 91 físicos médicos e 262 técnicos de radioterapia. Para os radioterapeutas e físicos, esses números não expressam a quantidade de profissionais em atividade, pois foi possível observar que um número expressivo deles tem vínculos com dois ou mais serviços, às vezes distantes entre si, o que contraria as normas.

\section{Perfis de desempenho das conformidades na proteção radiológica dos serviços de radioterapia}

Na Tabela 2, observa-se que as médias aritméticas das proporções de adequação (sendo $100,0 \%$ o valor a ser alcançado quando de acordo com os parâmetros estabelecidos) obtidas nos grupos e itens de conformidade pelo conjunto dos serviços no Estado de São Paulo (média total $=59,4 \%$, mediana $=59,6 \%$ ) oscilaram em uma faixa de valores entre $29,8 \%$ (cuidados/técnicas/programa de qualidade com pacientes) e 94,5\% (instalações físicas).

No Grupo Conformidades I, que teve adequação média geral de $61,1 \%$, o item plano de proteção radiológica apresentou a média nos serviços mais baixa $(45,0 \%)$, principalmente em decorrência da não atualização do plano junto à CNEN. No Grupo Conformidades II, com adequação média geral de $68,5 \%$, o item proteção radiológica das salas obteve a média nos serviços mais baixa $(45,9 \%)$, indicando dificuldades na rotina de manutenção de determinados quesitos de segurança, como mesas de comando e sistema de comunicação com os pacientes nas salas em funcionamento, ausência de sinalizações sobre a presença de radiação nas portas das salas de tratamento, portas com travas e maçanetas quebradas, ausência de monitores de área e dosímetros clínicos para medição da radiação, dentre outros. O item programa de garantia de qualidade (PGQ) apresentou uma variação entre os serviços de 35,7 a $100,0 \%$ (com média $=65,2 \%$ ), e os escores mais baixos foram decorrentes de interpretação inadequada ou desconhecimento de alguns serviços quanto às exigências estabelecidas em lei para o PGQ e relação paciente/máquina inadequada. Grande parte das máquinas de teleterapia funcionava em turnos prolongados, dificultando a realização de determinados testes na periodicidade adequada.

No Grupo conformidades III, que teve adequação média geral de $56,7 \%$, observou-se uma variação de adequação nos serviços com valores entre 20,0 e $80,0 \%$, sendo os baixos escores decorrentes das resistências dos funcionários ao uso de dosímetros e ausência de realização sistemática de exames laboratoriais. No Grupo conformidades $I V$, a adequação média geral foi de $51,4 \%$, a mais baixa dos quatro grupos, com variação das médias nos serviços de 33,3 a $74,4 \%$. Destaca-se o item cuidados com os pacientes com valores que variaram entre 0,0 e $73,3 \%$, observando-se inadequação nos procedimentos relativos à irradiação do tumor e minimização da irradiação às partes sãs, não disponibilidade de guias de condutas internas, ausência de monitoramento de condutas utilizadas e de doses aplicadas, e de análise ou monitoramento da efetividade dos tratamentos realizados. A média de adequação para o item procedimentos de emergência foi a segunda mais baixa $(43,9 \%)$ neste grupo, com ausência em alguns serviços de equipamentos e procedimentos de emergência em locais visíveis, botões de emergência e travas de portas com problemas, entre outros. No item preparo de equipe, com adequação média elevada $(80,6 \%)$, a não obtenção de escores satisfatórios em alguns serviços decorreu de problemas na capacitação da equipe e parâmetros inadequados na relação profissionais/máquina.

A classificação dos serviços de radioterapia em Baixa, Média e Alta conformidades foi feita com a distribuição em tercis dos valores mé- 
Tabela 2

Matriz de perfis de desempenho: proporções (\%) de conformidade dos serviços de radioterapia*. Estado de São Paulo, Brasil, 2000.

\begin{tabular}{|c|c|c|c|c|c|c|c|c|c|c|c|c|c|c|c|}
\hline \multirow{3}{*}{$\begin{array}{l}\text { Serviços } \\
\text { de radio- } \\
\text { terapia }\end{array}$} & \multirow{2}{*}{\multicolumn{4}{|c|}{ I - Documentação }} & \multicolumn{6}{|c|}{ Grupos de conformidades } & \multirow{2}{*}{\multicolumn{3}{|c|}{ IV - PR paciente }} & \multirow{3}{*}{$\begin{array}{l}\text { Média } \\
\text { total }\end{array}$} & \multirow{3}{*}{$\begin{array}{l}\text { Classifi- } \\
\text { cação } \\
\text { da con- } \\
\text { formi- } \\
\text { dade }{ }^{\star \star}\end{array}$} \\
\hline & & & & & $\begin{array}{l}\text { II - PR } \\
\text { vizin }\end{array}$ & $\begin{array}{l}\text { Júblico/ } \\
\text { anças }\end{array}$ & & & (n) & & & & & & \\
\hline & PPR & Outros & Média & $\begin{array}{l}\text { Inst. } \\
\text { físicas }\end{array}$ & $\begin{array}{l}\text { PR } \\
\text { salas }\end{array}$ & PGQ & Média & $\begin{array}{l}\text { Cuida- } \\
\text { dos }\end{array}$ & Média & Cd. pac. & $\begin{array}{l}\text { Pe- } \\
\text { merg. }\end{array}$ & Peq. & Média & & \\
\hline IHA39 & 15,8 & 64,3 & 40,0 & 66,7 & 33,3 & 42,9 & 47,6 & 50,0 & 50,0 & 33,3 & 20,0 & 62,5 & 38,6 & 44,1 & Baixa \\
\hline IHA22 & 10,5 & 85,7 & 48,1 & 100,0 & 33,3 & 42,9 & 58,7 & 40,0 & 40,0 & 33,3 & 30,0 & 37,5 & 33,6 & 45,1 & Baixa \\
\hline IHA36 & 47,4 & 42,9 & 45,1 & 100,0 & 33,3 & 64,3 & 65,9 & 40,0 & 40,0 & 6,7 & 20,0 & 75,0 & 33,9 & 46,2 & Baixa \\
\hline IHA37 & 47,4 & 50,0 & 48,7 & 100,0 & 33,3 & 64,3 & 65,9 & 40,0 & 40,0 & 6,7 & 20,0 & 75,0 & 33,9 & 47,1 & Baixa \\
\hline IHM29 & 36,8 & 42,9 & 39,9 & 100,0 & 26,7 & 64,3 & 63,7 & 40,0 & 40,0 & 26,7 & 40,0 & 87,5 & 51,4 & 48,7 & Baixa \\
\hline IHA27 & 31,6 & 71,9 & 51,5 & 100,0 & 40,0 & 57,1 & 65,7 & 40,0 & 40,0 & 26,7 & 30,0 & 62,5 & 39,7 & 49,2 & Baixa \\
\hline IHM34 & 31,6 & 57,1 & 44,4 & 100,0 & 46,7 & 57,1 & 67,9 & 50,0 & 50,0 & 13,3 & 40,0 & 62,5 & 38,6 & 50,2 & Baixa \\
\hline IAM38 & 31,6 & 50,0 & 40,8 & 100,0 & 40,0 & 42,9 & 61,0 & 60,0 & 60,0 & 0,0 & 20,0 & 100,0 & 40,0 & 50,4 & Baixa \\
\hline IHM40 & 31,6 & 78,6 & 55,1 & 66,7 & 26,7 & 57,1 & 50,2 & 50,0 & 50,0 & 26,7 & 40,0 & 75,0 & 47,2 & 50,6 & Baixa \\
\hline IAA31 & 31,6 & 71,4 & 51,5 & 100,0 & 26,7 & 57,1 & 61,3 & 50,0 & 50,0 & 13,3 & 50,0 & 62,5 & 42,0 & 51,2 & Baixa \\
\hline IHM42 & 21,0 & 92,9 & 57,0 & 100,0 & 46,7 & 57,1 & 67,9 & 50,0 & 50,0 & 13,3 & 40,0 & 50,0 & 34,4 & 52,3 & Baixa \\
\hline RHA11 & 26,3 & 85,7 & 56,0 & 66,7 & 33,3 & 42,9 & 47,6 & 80,0 & 80,0 & 20,0 & 30,0 & 50,0 & 33,3 & 54,2 & Baixa \\
\hline IHM30 & 26,3 & 50,0 & 38,2 & 100,0 & 40,0 & 71,4 & 70,5 & 50,0 & 50,0 & 20,0 & 60,0 & 100,0 & 60,0 & 54,7 & Baixa \\
\hline IHA43 & 15,8 & 92,9 & 54,3 & 100,0 & 53,3 & 50,0 & 67,8 & 60,0 & 60,0 & 20,0 & 30,0 & 75,0 & 41,7 & 55,9 & Baixa \\
\hline IAB45 & 26,3 & 78,6 & 52,5 & 100,0 & 66,7 & 64,3 & 77,0 & 20,0 & 20,0 & 53,3 & 70,0 & 100,0 & 74,4 & 56,0 & Baixa \\
\hline IHM33 & 31,6 & 78,6 & 55,1 & 100,0 & 33,3 & 71,4 & 68,3 & 50,0 & 50,0 & 13,3 & 40,0 & 100,0 & 51,1 & 56,1 & Baixa \\
\hline RAA18 & 42,1 & 92,9 & 67,5 & 33,3 & 46,7 & 71,4 & 50,5 & 50,0 & 50,0 & 46,7 & 40,0 & 87,5 & 58,1 & 56,5 & Média \\
\hline IAA28 & 36,8 & 64,3 & 50,6 & 100,0 & 33,3 & 64,3 & 65,9 & 60,0 & 60,0 & 13,3 & 40,0 & 100,0 & 51,1 & 56,9 & Média \\
\hline RHM14 & 52,6 & 92,9 & 72,8 & 100,0 & 40,0 & 42,9 & 60,9 & 60,0 & 60,0 & 0,0 & 40,0 & 62,5 & 34,2 & 57,0 & Média \\
\hline RHA3 & 68,4 & 50,0 & 59,2 & 66,7 & 40,0 & 100,0 & 68,9 & 50,0 & 50,0 & 46,7 & 30,0 & 75,0 & 50,6 & 57,2 & Média \\
\hline RHA7 & 26,3 & 71,4 & 48,9 & 100,0 & 33,3 & 78,6 & 70,6 & 50,0 & 50,0 & 33,3 & 60,0 & 87,5 & 60,3 & 57,5 & Média \\
\hline IHM20 & 31,6 & 78,6 & 55,1 & 100,0 & 46,7 & 57,2 & 67,9 & 60,0 & 60,0 & 40,0 & 40,0 & 62,5 & 47,5 & 57,6 & Média \\
\hline IHM24 & 26,3 & 92,9 & 59,6 & 100,0 & 46,7 & 71,4 & 72,7 & 50,0 & 50,0 & 20,0 & 40,0 & 87,5 & 49,2 & 57,9 & Média \\
\hline RAB19 & 31,6 & 50,0 & 40,8 & 100,0 & 80,0 & 85,7 & 88,6 & 30,0 & 30,0 & 53,3 & 70,0 & 100,0 & 74,4 & 58,5 & Média \\
\hline IAA46 & 52,6 & 100,0 & 76,3 & 100,0 & 46,7 & 64,3 & 70,3 & 50,0 & 50,0 & 13,3 & 50,0 & 62,5 & 41,9 & 59,6 & Média \\
\hline RHM15 & 26,3 & 71,4 & 48,9 & 100,0 & 46,7 & 78,6 & 75,1 & 60,0 & 60,0 & 26,7 & 40,0 & 100,0 & 55,6 & 59,9 & Média \\
\hline IAA41 & 31,6 & 78,6 & 55,1 & 100,0 & 33,3 & 71,4 & 68,3 & 60,0 & 60,0 & 20,0 & 50,0 & 100,0 & 56,7 & 60,0 & Média \\
\hline RHA16 & 47,4 & 57,1 & 52,3 & 66,7 & 46,7 & 57,1 & 56,8 & 80,0 & 80,0 & 26,7 & 70,0 & 62,5 & 53,1 & 60,5 & Média \\
\hline RHA12 & 52,6 & 78,6 & 65,6 & 100,0 & 33,3 & 57,1 & 63,5 & 70,0 & 70,0 & 20,0 & 50,0 & 62,5 & 44,2 & 60,8 & Média \\
\hline IHA25 & 42,1 & 92,9 & 67,5 & 100,0 & 40,0 & 71,4 & 70,5 & 70,0 & 70,0 & 26,7 & 30,0 & 50,0 & 35,6 & 60,9 & Média \\
\hline IHA21 & 42,1 & 71,4 & 56,8 & 100,0 & 33,3 & 78,6 & 70,6 & 60,0 & 60,0 & 20,0 & 50,0 & 100,0 & 56,7 & 61,0 & Média \\
\hline IHM26 & 42,1 & 92,9 & 67,5 & 100,0 & 40,0 & 78,6 & 72,9 & 50,0 & 50,0 & 33,3 & 30,0 & 100,0 & 54,4 & 61,2 & Média \\
\hline IAM47 & 68,4 & 71,4 & 69,9 & 100,0 & 53,3 & 42,9 & 65,4 & 80,0 & 80,0 & 6,7 & 10,0 & 87,5 & 34,7 & 62,5 & Alta \\
\hline IHM48 & 68,4 & 71,4 & 69,9 & 100,0 & 53,3 & 42,9 & 65,4 & 80,0 & 80,0 & 6,7 & 10,0 & 87,5 & 34,7 & 62,5 & Alta \\
\hline IHM35 & 100,0 & 85,7 & 92,9 & 100,0 & 46,7 & 64,3 & 70,3 & 50,0 & 50,0 & 20,0 & 40,0 & 62,5 & 40,8 & 63,5 & Alta \\
\hline RHA17 & 52,6 & 78,6 & 65,6 & 100,0 & 33,3 & 71,4 & 68,3 & 60,0 & 60,0 & 13,3 & 70,0 & 100,0 & 61,1 & 63,7 & Alta \\
\hline RHA6 & 36,8 & 78,6 & 57,7 & 100,0 & 40,0 & 85,7 & 75,2 & 60,0 & 60,0 & 60,0 & 40,0 & 100,0 & 66,7 & 64,9 & Alta \\
\hline RAA1 & 63,2 & 100,0 & 81,6 & 100,0 & 80,0 & 35,7 & 71,9 & 50,0 & 50,0 & 53,3 & 60,0 & 62,5 & 58,6 & 65,5 & Alta \\
\hline RHA13 & 68,4 & 42,9 & 55,6 & 100,0 & 80,0 & 92,9 & 90,9 & 70,0 & 70,0 & 33,3 & 60,0 & 62,5 & 51,9 & 67,1 & Alta \\
\hline IHB23 & 15,8 & 100,0 & 57,9 & 100,0 & 66,7 & 64,3 & 77,0 & 70,0 & 70,0 & 33,3 & 70,0 & 87,5 & 63,6 & 67,1 & Alta \\
\hline IHB32 & 31,6 & 100,0 & 65,8 & 100,0 & 73,3 & 71,4 & 81,6 & 50,0 & 50,0 & 53,3 & 60,0 & 100,0 & 71,1 & 67,1 & Alta \\
\hline RHA2 & 84,2 & 78,6 & 81,4 & 66,7 & 60,0 & 64,3 & 63,7 & 50,0 & 50,0 & 73,3 & 50,0 & 100,0 & 74,4 & 67,4 & Alta \\
\hline RHA8 & 73,7 & 92,9 & 83,3 & 100,0 & 46,7 & 64,3 & 70,3 & 70,0 & 70,0 & 60,0 & 40,0 & 62,5 & 54,2 & 69,4 & Alta \\
\hline RAB4 & 57,9 & 100,0 & 79,0 & 100,0 & 93,3 & 71,4 & 88,3 & 50,0 & 50,0 & 46,7 & 50,0 & 100,0 & 65,6 & 70,7 & Alta \\
\hline RHA10 & 63,2 & 92,9 & 78,0 & 100,0 & 46,7 & 92,9 & 79,8 & 70,0 & 70,0 & 33,3 & 60,0 & 87,5 & 60,3 & 72,0 & Alta \\
\hline IAA44 & 94,7 & 92,9 & 93,8 & 100,0 & 46,7 & 71,4 & 72,7 & 70,0 & 70,0 & 40,0 & 40,0 & 75,0 & 51,7 & 72,0 & Alta \\
\hline IHA49 & 73,7 & 100,0 & 86,8 & 100,0 & 33,3 & 64,3 & 65,9 & 70,0 & 70,0 & 46,7 & 50,0 & 100,0 & 65,6 & 72,1 & Alta \\
\hline RHA5 & 63,2 & 64,3 & 63,7 & 100,0 & 46,7 & 78,6 & 75,1 & 80,0 & 80,0 & 53,3 & 70,0 & 100,0 & 74,4 & 73,3 & Alta \\
\hline RHA9 & 73,7 & 100,0 & 86,8 & 100,0 & 46,7 & 78,6 & 75,1 & 70,0 & 70,0 & 60,0 & 60,0 & 100,0 & 73,3 & 76,3 & Alta \\
\hline $\begin{array}{l}\text { Média } \\
\text { total }\end{array}$ & 45,0 & 77,1 & 61,1 & 94,6 & 45,9 & 65,2 & 68,5 & 56,7 & 56,7 & 29,8 & 43,9 & 80,6 & 51,4 & 59,4 & \\
\hline $\begin{array}{l}\text { Pontos } \\
\text { máximos } \\
\text { brutos }\end{array}$ & 19 & 14 & & 3 & 15 & 14 & & 10 & & 15 & 10 & 4 & & & \\
\hline
\end{tabular}

* Dados referentes a 49 serviços de radioterapia participantes da pesquisa; ** Classificação de conformidade: baixa (de 44,1 a $56,1 \%$ ); média (de 56,5 a 61,2\%) e alta (de 62,5 a 76,3\%) (mediana = 59,6\%; desvio padrão = 7,94; desvio médio absoluto = 6,34).

PR = proteção radiológica; PPR = plano de proteção radiológica; Inst. físicas = instalações físicas; PGQ $=$ programa de garantia de qualidade:

Cd. pac. = cuidados com o paciente; Pemerg. = procedimentos de emergência; Peq. = preparo da equipe.

Região: $R$ = grande São Paulo; $I$ = interior. Condição: $A$ = unidade autônoma; $H$ = vinculada a hospital.

Complexidade tecnológica: $B$ = baixa; $M=$ média; $A$ = alta. 
dios gerais das proporções de adequação nos serviços, resultando nos valores 44,1 a $56,1 \%$ como Baixa conformidade, 56,5 a $61,2 \%$ como Média e 62,5 a 76,3\% como Alta conformidade (Tabela 2).

Todos os serviços de radioterapia classificados em alta conformidade apresentaram proporções de adequação acima de 50,0\% em todos os grupos de conformidade, exceto para o Grupo conformidades $I V$, em que três serviços estão abaixo dessa média (Tabela 3). Nos serviços de Média conformidade foram encontrados valores abaixo de 50,0\% em 3 serviços para o Grupo Conformidades I, um serviço para o Grupo Conformidades III e 6 serviços para o Grupo Conformidades IV (Tabela 3). Nos serviços de Baixa conformidade, proporções menores que $50,0 \%$ foram encontradas em 8 serviços para o Grupo Conformidades I, 2 para o Grupo Conformidades II, 6 para o Grupo Conformidades III e 12 para o Grupo Conformidades IV (Tabela 3$)$.

Dentre os 29 serviços de radioterapia considerados como de Alta complexidade, 11 foram classificados em Alta conformidade e destes, 9 estão localizados na capital, em hospitais considerados de primeira linha.

Os resultados obtidos apontaram importantes diferenças entre os estabelecimentos com relação ao atendimento às exigências regulatórias em cada grupo de conformidades e suas características gerais. Contudo, o item mais crítico para o conjunto dos serviços foi o Grupo Conformidades IV, no qual 21 (42,9\%) dos serviços avaliados apresentaram proporções de conformidades abaixo de 50,0\%.

A análise das normas técnicas e legislação vigentes e dos programas de controle sanitário mostra que há uma priorização de dimensões de estrutura, como o projeto de construção dos serviços e a descrição dos elementos que compõem o plano de proteção radiológica, bem como da documentação exigida para a obtenção de licenças de funcionamento do estabelecimento e das máquinas, controle e armazenamento das fontes de radiação, aspectos que inegavelmente representam condições básicas iniciais para a possibilidade de minimização dos riscos de exposição à radiação e proteção do público/vizinhanças, trabalhadores e pacientes. Contudo, a incorporação das normas dos programas de garantia de qualidade, que protegeriam mais diretamente os pacientes, é ainda incipiente na legislação nacional, embora ricas e detalhadas na legislação internacional. Na análise das inspeções realizadas pelos órgãos reguladores (CNEN e Vigilância Sanitária), ainda que se constatem diferenças nas suas formas de atuação, a serem aprofundadas em outro texto, foi possível observar que eram também priorizadas nas fiscalizações as dimensões estruturais.

\section{Conclusões}

Os resultados da pesquisa mostraram uma maior efetividade do programa no controle das conformidades com as dimensões estruturais, com valores mais elevados de adequação nos serviços, o que pode ser conseqüência da ênfase que elas têm na regulação sanitária nacional vigente. Os valores de adequação são mais baixos para os procedimentos relativos à rotina diária dependentes da conduta profissional e que demandam, para serem seguidos pelos serviços, uma maior freqüência de supervisões e fiscalização sanitária. A proteção radiológica do paciente se constituiu na dimensão mais crítica, com valores de adequação muito baixos em um grande número de serviços, sugerindo a necessidade de uma mudança na atuação das duas entidades responsáveis pela fiscalização dos serviços de radioterapia, CNEN e Vigilância Sanitária.

O estudo mostrou também que, apesar de se tratar de um setor altamente especializado e com elevada densidade tecnológica, há um processo predatório em curso pelo número insuficiente de serviços, excessiva demanda de pacientes e profissionais com múltiplas jornadas e responsabilidades técnicas, igualando-se nestes aspectos a outros setores especializados que compõem o sistema de saúde. Considerando-se que a maior parte dos serviços vinculase ao SUS, a metodologia desenvolvida para a avaliação das conformidades poderia ser de utilidade na atuação das entidades responsáveis pelo credenciamento e fiscalização dos serviços. A classificação em tipologias de conformidades Alta, Média e Baixa mostra-se com potencial utilidade também para o monitoramento dos serviços, tanto para o acompanhamento dos valores máximos que cada estabelecimento pode alcançar, quanto para identificar, no conjunto dos serviços, o impacto das políticas assistenciais no seu desempenho.

Os resultados encontrados colocam uma questão nuclear para a regulação sanitária em seu objetivo de prevenção de riscos e promoção da saúde: qual a relação entre as conformidades observadas, ou a ausência delas, e o risco sanitário? Que danos podem ocorrer ou estão ocorrendo em pacientes, trabalhadores e público/vizinhança nos serviços que funcionam com menos de 50,0\% dos itens em confor- 
Tabela 3

Distribuição dos serviços de radioterapia classificados na pesquisa como de Alta, Média e Baixa conformidades,

segundo intervalos de médias aritméticas obtidas (\% alcance) por grupos de conformidades no ano 2000.

\begin{tabular}{|c|c|c|c|c|c|c|c|c|c|c|}
\hline \multirow[t]{2}{*}{$\begin{array}{l}\text { Médias aritméticas } \\
\text { obtidas (\%) }\end{array}$} & \multicolumn{2}{|c|}{ I - Documentação } & \multicolumn{2}{|c|}{$\begin{array}{c}\text { II - Proteção } \\
\text { radiológica de } \\
\text { público/vizinhanças }\end{array}$} & \multicolumn{2}{|c|}{$\begin{array}{l}\text { III - Proteção } \\
\text { radiológica dos } \\
\text { trabalhadores }\end{array}$} & \multicolumn{2}{|c|}{$\begin{array}{l}\text { IV - Proteção } \\
\text { radiológica do } \\
\text { paciente }\end{array}$} & \multicolumn{2}{|c|}{ Média total } \\
\hline & $\mathrm{n}$ & $\%$ & $\mathrm{n}$ & $\%$ & $n$ & $\%$ & n & $\%$ & $\mathrm{n}$ & $\%$ \\
\hline \multicolumn{11}{|l|}{ Alta conformidade } \\
\hline $20 \vdash 30$ & 0 & 0,0 & 0 & 0,0 & 0 & 0,0 & 0 & 0,0 & 0 & 0,0 \\
\hline $30 \vdash 40$ & 0 & 0,0 & 0 & 0,0 & 0 & 0,0 & 2 & 11,8 & 0 & 0,0 \\
\hline $40 \vdash 50$ & 0 & 0,0 & 0 & 0,0 & 0 & 0,0 & 1 & 5,9 & 0 & 0,0 \\
\hline Subtotal & 0 & 0,0 & 0 & 0,0 & 0 & 0,0 & 3 & 17,7 & 0 & 0,0 \\
\hline $50 \vdash 60$ & 3 & 17,6 & 0 & 0,0 & 5 & 29,4 & 4 & 23,5 & 0 & 0,0 \\
\hline $60+70$ & 5 & 29,4 & 5 & 29,4 & 2 & 11,8 & 6 & 35,3 & 11 & 64,7 \\
\hline Subtotal & 8 & 47,0 & 5 & 29,4 & 7 & 41,2 & 10 & 58,8 & 11 & 64,7 \\
\hline $70 \vdash 80$ & 2 & 11,8 & 9 & 52,9 & 7 & 41,2 & 4 & 23,5 & 6 & 35,3 \\
\hline $80 \vdash 90$ & 5 & 29,4 & 2 & 11,8 & 3 & 17,6 & 0 & 0,0 & 0 & 0,0 \\
\hline $90 \vdash 100$ & 2 & 11,8 & 1 & 5,9 & 0 & 0,0 & 0 & 0,0 & 0 & 0,0 \\
\hline Subtotal & 9 & 53,0 & 12 & 70,6 & 10 & 58,8 & 4 & 23,5 & 6 & 35,3 \\
\hline Total & 17 & 100,0 & 17 & 100,0 & 17 & 100,0 & 17 & 100,0 & 17 & 100,0 \\
\hline \multicolumn{11}{|l|}{ Média conformidade } \\
\hline $20 \vdash 30$ & 0 & 0,0 & 0 & 0,0 & 0 & 0,0 & 0 & 0,0 & 0 & 0,0 \\
\hline $30 \vdash 40$ & 0 & 0,0 & 0 & 0,0 & 1 & 6,2 & 2 & 12,5 & 0 & 0,0 \\
\hline $40 \vdash 50$ & 3 & 18,7 & 0 & 0,0 & 0 & 0,0 & 4 & 25,0 & 0 & 0,0 \\
\hline Subtotal & 3 & 18,7 & 0 & 0,0 & 1 & 6,2 & 6 & 37,5 & 0 & 0,0 \\
\hline $50 \vdash 60$ & 7 & 43,7 & 2 & 12,5 & 6 & 37,5 & 8 & 50,0 & 10 & 62,5 \\
\hline $60+70$ & 4 & 25,0 & 6 & 37,5 & 6 & 37,5 & 1 & 6,2 & 6 & 37,5 \\
\hline Subtotal & 11 & 68,7 & 8 & 50,0 & 12 & 75,0 & 9 & 56,2 & 16 & 100,0 \\
\hline $70 \vdash 80$ & 2 & 12,5 & 7 & 43,7 & 2 & 12,5 & 1 & 6,2 & 0 & 0,0 \\
\hline $80 \vdash 90$ & 0 & 0,0 & 1 & 6,2 & 1 & 6,2 & 0 & 0,0 & 0 & 0,0 \\
\hline $90 \vdash 100$ & 0 & 0,0 & 0 & 0,0 & 0 & 0,0 & 0 & 0,0 & 0 & 0,0 \\
\hline Subtotal & 2 & 12,5 & 8 & 50,0 & 3 & 18,7 & 1 & 6,2 & 0 & 0,0 \\
\hline Total & 16 & 100,0 & 16 & 100,0 & 16 & 100,0 & 16 & 100,0 & 16 & 100,0 \\
\hline \multicolumn{11}{|l|}{ Baixa conformidade } \\
\hline $20 \vdash 30$ & 0 & 0,0 & 0 & 0,0 & 1 & 6,2 & 0 & 0,0 & 0 & 0,0 \\
\hline $30 \vdash 40$ & 2 & 12,5 & 0 & 0,0 & 0 & 0,0 & 8 & 50,0 & 0 & 0,0 \\
\hline $40 \vdash 50$ & 6 & 37,5 & 2 & 12,5 & 5 & 31,2 & 4 & 25,0 & 6 & 37,5 \\
\hline Subtotal & 8 & 50,0 & 2 & 12,5 & 6 & 37,4 & 12 & 75,0 & 6 & 37,5 \\
\hline $50 \vdash 60$ & 8 & 50,0 & 2 & 12,5 & 7 & 43,7 & 2 & 12,5 & 10 & 62,5 \\
\hline $60 \vdash 70$ & 0 & 0,0 & 10 & 62,5 & 2 & 12,5 & 1 & 6,2 & 0 & 0,0 \\
\hline Subtotal & 8 & 50,0 & 12 & 75,0 & 9 & 56,2 & 3 & 18,7 & 10 & 62,5 \\
\hline $70 \vdash 80$ & 0 & 0,0 & 2 & 12,5 & 0 & 0,0 & 1 & 6,2 & 0 & 0,0 \\
\hline $80 \vdash 90$ & 0 & 0,0 & 0 & 0,0 & 1 & 6,2 & 0 & 0,0 & 0 & 0,0 \\
\hline $90 \vdash 100$ & 0 & 0,0 & 0 & 0,0 & 0 & 0,0 & 0 & 0,0 & 0 & 0,0 \\
\hline Subtotal & 0 & 0,0 & 2 & 12,5 & 1 & 6,2 & 1 & 6,2 & 0 & 0,0 \\
\hline Total & 16 & 100,0 & 16 & 100,0 & 16 & 100,0 & 16 & 100,0 & 16 & 100,0 \\
\hline
\end{tabular}


midade com a legislação? Essas questões indicam a necessidade de estudos adicionais, para que se possa verificar se o controle sanitário proposto (porém exercido de forma incompleta), que se apóia fundamentalmente na análise de dimensões de estrutura e processo nos serviços, permite efetivamente a obtenção dos resultados desejados, a garantia da saúde da população.

\section{Resumo}

Os serviços de radioterapia respondem por procedimentos terapêticos essenciais para o câncer, uma das principais causas de morbidade e mortalidade populacional. Apesar de sua importância no sistema de saúde e do seu potencial de risco pelo uso da radiação ionizante, poucos estudos têm sido dedicados a eles. Neste estudo foram avaliadas as conformidades às normas técnicas de proteção radiológica dos serviços de radioterapia do Estado de São Paulo, Brasil. Foram estudados 49 serviços de radioterapia em 2000, por meio de entrevistas com seus responsáveis. Foram desenvolvidas tipologias de desempenho baseadas em variáveis de estrutura e processo, e realizada análise comparativa dos resultados encontrados nos serviços. Foram observadas diferenças importantes nos serviços quanto à posição no sistema de saúde, nível de complexidade e distribuição geográfica, com desempenho médio melhor para as condições estruturais e desempenho muito inadequado na proteção ao paciente, apontando para a necessidade de uma vigilância sanitária mais efetiva.

Neoplasia; Radioterapia; Proteção Radiológica; Vigilância Sanitária

\section{Colaboradores}

M. B. P. Eduardo e H. M. D. Novaes, em conjunto, conceberam e planejaram o artigo, realizaram discussão e aprimoramento de frases e principais dados do enfoque do texto a serem divulgados. M. B. P. Eduardo redigiu o trabalho e ambas as autoras realizaram sua revisão final.

\section{Referências}

1. Borras C. Organization, development, quality assurance and radiation protection in radiology services: imaging and radiation therapy. Washington DC: Pan American Health Organization, World Health Organization; 1997.

2. Secretaria de Estado da Saúde do Estado de São Paulo. Mortalidade por câncer no Estado de São Paulo 1988-1998. São Paulo: Fundação Oncocentro de São Paulo, Secretaria de Estado da Saúde do Estado de São Paulo; 2000.

3. Comissão Nacional de Energia Nuclear. Solicitação de concessão de licenças e autorizações/ cadastros. Rio de Janeiro: Centro de Informações Nucleares, Comissão Nacional de Energia Nuclear; 2000. (Documento técnico).

4. Instituto Nacional de Câncer. Estimativas da incidência e mortalidade por câncer no Brasil para 1998. Rio de Janeiro: Instituto Nacional de Câncer; 1998.

5. Scaff LAM. Física da radioterapia. São Paulo: Sarvier; 1997.

6. International Atomic Energy Agency. Design and implementation of a radiotherapy programme: clinical, medical physics, radioation protection and safety aspects. Vienna: International Atomic Energy Agency; 1998.

7. Koukoulias VE, Poortmans PM, Bernier J, Horiot JC, Johansson KA, Davis B, et al. The Quality Assurance programme of Radiotherapy Group of the European Organization for Research and Treatment of Cancer (EORTC): a critical appraisal of 20 years of continuous efforts. Eur J Cancer 2003; 39:430-7.

8. Koukoulias VE. Quality assurance in radiothrerapy. Eur J Cancer 2003; 39:415-22. 
9. Dixon P, O'Sullivan B. Radiotherapy quality assurance: time for everyone t take it seriously. Eur J Cancer 2003; 39:423-9.

10. Kneebone A, Turner S, Berry M, Cakir B, Gebski V. Australian prostate-specific antigen outcome and toxicity following radiation therapy for localized prostate cancer. Australas Radiol 2003; 47:422-7.

11. Williams J, Chen Y, Rubin P, Finkelstein J, Okunieff $P$. The biological basis of a comprehensive grading system for the adverse effects of cancer treatment. Semin Radiat Oncol 2003; 13:182-8.

12. Eich HT, Muller RP, Schneeweiss A, Hansemann $\mathrm{K}$, Semrau R, Willich N, et al. Initiation of a teleradiotherapeutic network for patients in German lymphoma studies. Int J Radiat Oncol Biol Phys 2004; 58:805-8.

13. Aspden P, Corrigan JM, Wolcott J, Erickson SM. Patient safety: achieving a new standard for care. Washington DC: The National Academy of Medicine Press; 2004.

14. Shrimpton PC. The role of United Nations Scientific Committee on the effects of atomic radiation in relation to medical exposures. In: Proceedings of the International conference on radiological protection of patients in diagnostic and interventional radiology, nuclear medicine and radiotherapy. Malaga: International Atomic Energy Agency; 2001. p. 71-6.

15. Ortiz-Lopez P. Role of the IAEA in the radiological protection of patients. In: Proceedings of the International conference on radiological protection of patients in diagnostic and interventional radiology, nuclear medicine and radiotherapy. Malaga: International Atomic Energy Agency; 2001. p. 35-46.

16. Vitoria AH, Gonzalez BF, Climent JM, Calatayud JP. Consequences and problems which arose from the applications of the Spanish laws about quality criteria in radiodiagnostics, nuclear medicine and radiotherapy from the point of view of radiophysicists. In: Proceedings of the International Conference on Radiological Protection of Patients in Diagnostic and Interventional Radiology, Nuclear Medicine and Radiotherapy. Malaga: International Atomic Energy Agency; 2001. p. 31-4.

17. Esco R, Pardo J, Palacios A, Biete A, Fernandez J, Valls A, et al. Final report from the Spanish Society of Radiotherapy and Oncology Infrastructures Commission about Department Standards Recommendable in Radiation Oncology. In: Proceedings of the International Conference on Radiological Protection of Patients in Diagnostic and Interventional Radiology, Nuclear Medicine and Radiotherapy. Malaga: International Atomic Energy Agency; 2001. p. 77-81.

18. Department of Human Services. Review of radiotherapy services Victoria, Australia: discussion paper and submission guide, overview and recommendations. Victoria: Department of Human Services; 1997. (Technical document).

19. Hummel S, Paisley S, Morgan A, Currie E, Brewer N. Clinical and cost-effectiveness of new and emerging technologies for early localized prostate cancer: a systematic review. Health Technol Assess 2003; 7:1-157.

20. Barton MB, Peters LJ, Kenny LM. Radiotherapy in
Australia one year after the Baume report: vison or mirage? Med J Aust 2004; 180:55-6.

21. Thomas SJ. Capacity and demand models for radiotherapy treatment machines. Clin Oncol 2003; 15:353-8.

22. Slotman BJ, Leer JW. Infrastructure of radiotherapy in the Netherlands: evaluation of prognosis and introduction of a new model for determining the needs. Radiother Oncol 2003; 66:345-9.

23. De Cuyper L, Van Hee R, Sterckx F. The effect of social factors on diagnosis and treatment of breast cancer. Acta Chir Belg 2003; 103:585-8.

24. Aldred MA, Furnari L, Eduardo MBP. Elaboração, publicação e implementação de uma norma técnica de proteção radiológica no Estado de São Paulo. In: Schiabel H, Slaets AFF, Costa LF, Baffa Filho O, Marques PMA, organizadores. Anais do III Fórum Nacional de Ciência e Tecnologia em Saúde. São Carlos: Sociedade Brasileira de Engenharia Biomédica; 1996. p. 483-4.

25. Calcina CSG, Almeida A, Rocha JRO. Análises de protocolos de braquiterapia, por alta taxa de dose, do controle de qualidade de alguns serviços locais, baseados no TG40, TG56 e ARCAL XXX. Radiol Bras 2001; 34:225-32.

26. Calcina CSG, Lima LP, Rubo RA. Análises de protocolos teleterápicos de controle de qualidade de alguns serviços locais, baseados no TG40 e ARCAL XXX. Radiol Bras 2002; 35:31-40.

27. Cecílio PJ. Emergências em braquiterapia de alta taxa de dose: manual de conduta. Radiol Bras 2001; 34:39-43.

28. Souza CN, Monti CR, Sibata CH. Recomendações para se evitar grandes erros de dose em tratamentos radioterapêuticos. Radiol Bras 2001; 34:29-37.

29. Canary PC, Almeida CE. A radioterapia do colo do útero no Brasil. Rev Bras Cancerol 1998; 44:101-7.

30. Faria SL. Radioterapia em linfomas não-Hodgkin. Rev Bras Cancerol 1992; 38(2/3):91-7.

31. Zanardo MLAM. Distribuição de recursos de alta tecnologia: a situação da radioterapia [Dissertação de Mestrado]. São Paulo: Escola de Administração de Empresas de São Paulo; 1992.

32. Drexler P, Cunha PG, Peixoto JE. Medical and occupational exposures in Brasil. Radiat Prot Dosimetry 1991; 36:101-5.

33. Novaes HDM. Avaliação de programas, serviços e tecnologia em saúde. Rev Saúde Pública 2000; 34:547-9.

34. Clarke A. Evaluation research: an introduction to principles. Methods and practice. London: Sage Publications; 1999.

35. Patton MQ. Utilization-focused evaluation: the new century text. 3rd Ed. London: Sage Publications; 1997.

36. Mertens DM. Inclusive evaluation: implications of transformative theory for evaluation. American Journal of Evaluation 1999; 20:1-4.

37. Centers for Disease Control and Prevention. Framework for program evaluation in public health - recommendations and reports. MMWR Morb Mortal Wkly Rep 1999; 48:1-40.

38. Donabedian A. The seven pillars of quality. Arch Pathol Lab Med 1990; 114:1115-8.

39. Joint Committee on Standards for Educational Evaluation. The program evaluation standards: 
how to assess evaluations of educational programs. 2nd Ed. London: Sage Publications; 1994.

40. Comissão Nacional de Energia Nuclear. NE - 6.02: dispõe sobre o licenciamento de instalações radiativas. CNEN. DNE-21, Res. 9/84. Diário Oficial da União 1984; 16 dez.

41. Comissão Nacional de Energia Nuclear. NE - 3.01: dispõe sobre as diretrizes básicas de radioproteção. CNEN. DNE-32, Res. 12/88. Diário Oficial da União 1988; 01 ago.

42. Comissão Nacional de Energia Nuclear. NE - 3.02: dispõe sobre serviços de radioproteção. CNEN. DNE-33, Res. 10/88. Diário Oficial da União 1988; 01 ago.

43. Comissão Nacional de Energia Nuclear. NE - 3.06: estabelece requisitos de radioproteção e segurança para serviços de radioterapia. CNEN. DNE43, Res. 01/90. Diário Oficial da União 1990; 30 mar.

44. Resolução CNS n. 06, de 21 de dezembro de 1988. Aprova as normas técnicas gerais de radioproteção para a defesa da saúde dos pacientes, indivíduos profissionalmente exposto e do público em geral, para cumprimento do disposto no art. 9o do Decreto n. 81.384, de 22 de fevereiro de 1978. Diário Oficial da União 1989; 05 jan.
45. Resolução SS n. 625, de 14 de maio de 1994. Aprova norma técnica que dispõe sobre o uso, posse e armazenamento de fonte de radiação ionizante no âmbito do Estado de São Paulo. Diário Oficial do Estado de São Paulo 1994; 15 dez.

46. Decreto n. 45.615, de 04 de janeiro de 2001. Dispõe sobre a concessão de licenças de funcionamento, certificados de vistoria sanitária, cadernetas de controle sanitário, alvarás de utilização e dá providências correlatas. Diário Oficial do Estado de São Paulo 2001; 05 jan.

47. Portaria GM/MS n. 3.535, de 02 de setembro de 1998. Estabelece critérios para cadastramento de centros de atendimento em oncologia. Diário Oficial da União 1998; 03 set.

48. Portaria MS n. 113, de 31 de março de 1999. Estabelece normas para cadastramento dos serviços que executam procedimentos de alta complexidade em câncer. Diário Oficial da União 1999; 01 abr.

49. Gottfried KLD, Penn G, editors. Radiation in medicine: a need for regulatory reform. Washington DC: Institute of Medicine/The National Academy Press; 1996.

Recebido em 21/Jan/2004

Versão final reapresentada em 26/Jul/2004 Aprovado em 29/Jul/2004 